\title{
Hot and Cool Executive Function in Children and Adolescents with Autism Spectrum Disorder: Cross Sectional Developmental Trajectories
}

\author{
E. Kouklari, S. Tsermentseli, \& C. P. Monks \\ Accepted for publication: Child Neuropsychology
}

\begin{abstract}
The development of executive function (EF) in autism spectrum disorder (ASD) has only been investigated using "cool"-cognitive- EF tasks. Little is known about the development of "hot"affective- EF and whether it follows a similar developmental pathway. This study employed a cross-sectional developmental trajectories approach to examine the developmental changes in cool (working memory, inhibition, planning) and hot EF (delay discounting, affective decision making) of ASD participants $(\mathrm{n}=79)$ and controls $(\mathrm{n}=91)$ relative to age and IQ, shedding more light on the hot-cool EF organisation. The developmental trajectories of some aspects of cool EF (working memory, planning) differed significantly as a function of age in ASD participants relative to controls. For both hot EFs no significant age-related changes were found in either group. These findings extend our understanding regarding the maturation of EF from childhood through adolescence in ASD.
\end{abstract}

Keywords: ASD • hot \& cool Executive function • Trajectories $\bullet$ School Age 
HOT \& COOL EF TRAJECTORIES IN ASD

\section{Introduction}

Impaired Executive Function $(\mathrm{EF})$ has been identified as a salient characteristic across several Autism Spectrum Disorder (ASD) samples (Hill, 2004). EF refers to a set of future-oriented and goal-directed cognitive skills that are crucial for problem solving and social behaviour, as well as the ability to organise oneself (Anderson, 1998). The complex, multifaceted EF construct emerges in the early years of life, followed by critical changes throughout the preschool period. Maturation is reached in adolescence, protracted to the developmental course of the prefrontal cortex (underlying neural base) (Anderson, 1998). Despite the diversity of the EF construct, supported by imaging evidence showing that different EF tasks activate different areas of the prefrontal cortex (Gilbert \& Burgess, 2008; Wagner et al., 2001), most studies in ASD and typical development have viewed EF mainly through a purely cognitive lens, considered to be elicited under relatively abstract, non-affective conditions. However, developmentalists suggest that EF should be conceptualized as a broader construct, as it includes affective control processes as well ("hot processes"; Zelazo \& Müller, 2002). Metcalfe and Mischel (1999) were among the first to introduce the "hot-cool systems" distinction, proposing that hot processes are emotional influences on behaviour controlled by cool EF processes. Building on this initial model, Zelazo and Müller (2002) suggested a similar yet fundamentally different construct of EF that considers "hot" and "cool" processes as two distinct domains. Hot EF processes differ from cool EF processes but may coordinate with them, according to each task's demands. Hot EF refers to top-down processes operating in emotional or motivationally significant situations (hot tasks), such as delay discounting (i.e., the tendency to choose more immediate, smaller rewards) and affective decision making (i.e., mental processing occurring in the selection of one or more possible options under risk where 
HOT \& COOL EF TRAJECTORIES IN ASD

one employs both rational and emotional processes). In contrast, cool EF aspects involve topdown processes elicited in affectively neutral, nonaffective contexts (cool tasks), (Zelazo \& Carlson, 2012), including inhibition, working memory, or planning. Relative to the abstract, decontextualized cool EF tasks, hot EF tasks have meaningful rewards and losses for the participants; this affective salience of the situation and the associated cognitive processes are what distinguish hot from cool EF tasks (i.e. gambling tasks, the Marshmallow Test, or delay discounting tasks) (Zelazo \& Carlson, 2012). Hot and cool EF have been found to be dissociated in lesioned brains, but it is generally argued that they typically work together as part of a more general adaptive function (Zelazo \& Müller, 2002). Zelazo and Müller's (2002) hot and cool EF distinction model was employed in this study, as it has been proposed to shed more light on the roles of specific EF deficits in developmental disorders (Zelazo \& Carlson, 2012). Despite research supporting separate domains of cool and hot EF (Kim et al., 2014; Willoughby et al., 2011), traditionally, the development of EF has been investigated mainly using cool EF tasks which lack significant emotional components. Thus, little is known about the developmental trajectories of "hot"-affective- EF processes and whether cool and hot EF follow a similar developmental pathway in ASD and in typical development.

In Best and Miller's (2010) excellent review of the typical development of EF across childhood and adolescence, the developmental pattern of cool EF inhibition is suggested as showing rapid improvements between the $3^{\text {rd }}$ and $5^{\text {th }}$ year. By the age of 4 years, children are able to successfully perform tasks such as response inhibition (i.e. Go/No-Go), followed by less dramatic advances during middle childhood. Improvements are present between 5 and 8 years of life (Romine \& Reynolds, 2005) as well as beyond 10 years (more subtle), especially on computerized tasks such as Go/No-Go tasks, with slower increases during adolescence. The performance improvements of later childhood seem to involve mainly quantitative gains in 
HOT \& COOL EF TRAJECTORIES IN ASD

accuracy, as they more likely reflect an increasing capacity of children to override prepotent responses (Best \& Miller, 2010). Several, possibly overlapping areas of the prefrontal cortex, including the dorsal areas of the lateral prefrontal cortex and the anterior cingulate cortex and inferior frontal gyrus are suggested to interact in order to facilitate inhibition task performance (Duncan \& Owen, 2000; Konishi, Jimura, Asari, \& Miyashita, 2003). Despite age-related improvements in task performance often being subtle across middle childhood and adolescence, the underlying neural activity changes more dramatically (Johnstone et al., 2007), with greater brain localization and efficient activation in the aforementioned brain regions, pertinent to task completion (Best \& Miller, 2010)

Unlike inhibition that presents the most crucial improvements in preschool years and less dramatic changes throughout middle childhood/ adolescence, the developmental trajectory of working memory demonstrates linear increases from preschool age to adolescence. Most working memory tasks present performance improvements throughout the preschool period (Garon et al., 2008) while working memory ability is thought to have been sufficiently developed by the age of 6 , in order to be used in more complex working memory tasks (Gathercole et al., 2004). Both simple and complex working memory tasks (i.e. tasks that require coordination of several working memory subcomponents) demonstrate similar developmental courses of performance gains between the $4^{\text {th }}$ and $15^{\text {th }}$ year of life. Neuroimaging evidence has shown that the neural basis underpinning working memory is the dorsolateral prefrontal cortex (Best \& Miller, 2010; Funahashi, 2004), with the left temporofrontal cortex specifically found to be implicated in verbal working memory tasks (Thomason et al., 2009). The protracted neural developmental trajectory of working memory involves regressive and progressive alterations and leads to localized activity within the prefrontal cortex network of connectivity (Best \& Miller, 2010). 
HOT \& COOL EF TRAJECTORIES IN ASD

Finally, regarding the development of planning, evidence has demonstrated poor performance in early childhood with age-related improvements across middle childhood and adolescence (11-14 years); the age of 12 is suggested to be the age point of effective planning skills (De Luca, 2003). Studies which used different versions of the Tower of London task (Shallice, 1982) to examine age-related improvements in planning, have mainly reported significant developmental changes through middle childhood and early adolescence (5-12 years) (Korkman et al., 2001) as well as fewer errors made until late adolescence (Albert \& Steinberg, 2011; Asato et al., 2006; Huizinga et al., 2006). Generally, the performance on planning measures is thought to reach a plateau between the ages of 15 and 30 years (Anderson et al., 2001; De Luca et al., 2003). Evidence from imaging studies suggests that the activated brain areas during planning tasks are localized in the circumscribed neural assemblies of the middorsolateral part of the prefrontal cortex (Bechara et al., 2000; Manes et al., 2002; Unterrainer \& Owen, 2006).

Relative to cool EF tasks that mainly rely on the dorsal and lateral prefrontal cortex, as described above, evidence from lesion studies and Krain et al.'s (2006) meta-analysis has shown that particular hot $\mathrm{EF}$ aspects (i.e. affective decision-making) may be underpinned by different areas such as the orbitofrontal and ventromedial regions of the prefrontal cortex (Bechara, 2004). These regions are also connected with the amygdala and limbic system that underlie emotional processing (Phan, Wager, Taylor, \& Liberzon, 2004). Regarding the development of hot $\mathrm{EF}$, there has been very little research to date; it is thought that hot $\mathrm{EF}$ follows a rapid development during the preschool years in typical development (Zelazo \& Müller, 2002) and that age-related improvements are demonstrated during middle childhood and adolescence (Prencipe et al., 2011). Empirical findings (Kim et al., 2014; Willoughby et 
HOT \& COOL EF TRAJECTORIES IN ASD

al., 2011) and the afore-mentioned neuroimaging evidence posit that cool and hot EF are regulated by independent constructs, results about their developmental courses in typical development are mixed. Hongwanishkul et al. (2005) investigated the development of cool and hot $\mathrm{EF}$ in preschoolers (3-5 years) and reported no significant differences in the development of the two domains. Hot and cool EF measures were significantly correlated and presented performance gains after the third year. Willoughby et al. (2011) also found that cool and hot EF tasks were positively correlated in young children (3-5 years) providing evidence for nondistinct cool and hot EF constructs. Beyond the preschool period, Hooper et al. (2004) used two measures of cool EF (Go/No-Go task, Digit Span) and one hot (Iowa Gambling Task) with children and young people aged 9-17 years and reported age-related improvements on all tasks. Building on Hooper et al.'s study (2004), Prencipe et al. (2011) assessed typically developing children and adolescents (8-15 years) and indicated that performance reached mature levels at an older age on the hot EF tasks relative to cool EF measures. In addition, for both studies, only weak correlations were reported among hot and cool EF measures. Taking these findings together, it seems that hot and cool EF are likely to develop independently into adolescence, with hot EF possibly following a differentiated developmental trajectory beyond 5 years of age.

It is unclear whether the developmental trajectory of cool and hot EF in ASD is similar to that identified in typical development, prompting questions about the nature of the executive dysfunction in ASD. Evidence from previous neuroimaging studies in ASD in several brain areas, including the frontal cortex, have documented atypical patterns of white and grey matter volumes (Carper \& Courchesne, 2005; Mak-Fan et al., 2012), functional connectivity (Just et al., 2004; Koshino et al., 2005) and brain lateralization (McPartland et al., 2004) relative to typical development. Despite the substantial evidence for abnormalities in the development of the frontal lobes within the autism spectrum, the associations of these circuits with cognitive performance/ behavioural phenomena are not clear (Griebling et al., 2010). It remains to be 
HOT \& COOL EF TRAJECTORIES IN ASD

investigated whether EF impairments in ASD arise from prefrontal cortex deficits (i.e. deficient connectivity) or other underlying system impairment such as maturation (i.e. myelination changes) across development. Although the present study did not include neuroimaging or electrophysiological data, we strongly believe that developmental studies with broad age ranges could provide a solid ground to clarify, theoretically first, whether there is a developmental delay, deviance, or the deficits are constant across development in ASD.

The cross-sectional and few longitudinal studies investigating EF chronological age-related changes in school-aged children with ASD have focused only on cool aspects yielding inconsistent results. More specifically, some of these have reported age-related gains in ASD samples across middle childhood and adolescence (8-18 years) in aspects such as inhibition, and working memory (Happé et al., 2006a; Van Eylen et al., 2015) as well as planning (Happé et al., 2006a), suggesting that EF performance may improve with age in individuals with high functioning ASD. Inconsistent with the aforementioned studies, Luna et al.'s (2007) study, also using cross-sectional design with three age samples (8-12 years, 13-17 years, 18-33 years) reported deficits in cool EF inhibition for the ASD group across development. Furthermore, Ozonoff et al. (2004) in their broad age-range study (6-47 years) showed that there was no significant correlation between age and performance on EF planning. The afore-mentioned cross-sectional studies used traditional matched-group comparisons that do not allow for drawing conclusions regarding the continuous change of EF over the course of development. Ozonoff and McEvoy (1994) longitudinally assessed children and adolescents with ASD in cool EF measures of planning and cognitive flexibility first at 12 years and then at 15 years of age (3 year follow-up study) reporting a lack of age-related improvements. Results showed that the ASD participants demonstrated significantly poorer performance, which appeared to reach a developmental ceiling compared to the control group (peers without ASD but with learning 
HOT \& COOL EF TRAJECTORIES IN ASD

disabilities). A similar study in the preschool period (Pellicano, 2010) followed children with ASD (mean age 5.5 years) for 3 years measuring performance in the cool EF aspects of set shifting and planning. Results showed that planning in ASD improved significantly over the 3year period, surprisingly at an even faster rate relative to controls. Finally, the few studies that investigated the development of cool EF measured by rating scales (BRIEF; working memory, inhibition, shift, and planning) in ASD have also yielded inconsistent results. Rosenthal et al. (2013) showed that working memory performance was poorer in older participants with ASD (14-18 years) compared to the younger ones (6-7 years) implying that deficits in working memory increase in adolescents with ASD as reported by parents. No age-related improvements were found in the remaining subscales. In a similar developmental study with children and adolescents with ASD (6-18 years), using the same BRIEF subscales, Van den Bergh et al. (2014) found that inhibition presented age-related improvements while planning deficits were more evident in older participants relative to younger ones. No age-related improvements were found in working memory of children and adolescents with ASD.

No study to date has investigated the development of hot EF in ASD and thus relatively little is known about its developmental trajectory within ASD. As already discussed, investigating developmental trends of EF in ASD is important to provide a better insight into the brain maturation mechanisms in ASD and may shed light on potential implications for treatment. Moreover, there is no study to date having explored whether cool and hot EF subcomponents are distinct in ASD. Making speculations about the distinction of hot and cool EF in ASD is hard as there is no empirical evidence. For example, brain activation/ localization during distinct EF tasks has not been examined in ASD (Hill \& Frith 2003). Potential differences in the developmental trajectories of hot and cool EF within broad age ranges is a "hot", open topic of debate that the present study aims to address. Such data would be crucial to identify the 
HOT \& COOL EF TRAJECTORIES IN ASD

organisation and developmental relationship of cool and hot EF skills that could aid in overcoming the limitations of current theories of EF development and lead to a better understanding of the heterogeneity in neurocognitive impairments in ASD.

The main goal of this study was to investigate whether the developmental trajectories of hot and cool EF relative to chronological age and IQ differ between children and adolescents with autism and a typically developing group. IQ was included in analysis as previous studies have revealed a significant relation between cool EF and IQ, suggesting that higher IQ scores are associated with better performance in cool $\mathrm{EF}$ abilities on a variety of different measures (Arffa, 2007; Pellicano, 2007; Van Eylen et al., 2015). Taken together, previous studies (described above) show there is no clear developmental framework of cool EF in ASD due to inconsistent results; with some reporting age-related improvements and others not. Moreover, to date no research has investigated the development of hot EF in ASD. Employing tasks to assess both cool and hot EF skills, the current study will shed more light on the developmental pathway of EF followed in ASD across childhood and adolescence. Instead of the traditional group comparison, we used a cross-sectional developmental trajectories approach (Thomas et al., 2009) that uses cross-sectional data to explore developmental relationships by focusing on changes in domains of interest. Contrary to testing differences in cross-sectional group means, which masks changes associated with age or other foundational cognitive abilities, the trajectories approach evaluates group differences with respect to two coefficients, the intercepts and slopes of development. This methodology could reveal important information about the nature of development as it identifies not only early onset but also slower or deviant rates of development. However, to date no research has used this as a method of understanding whether the development of EF in ASD, fractionated into hot and cool subcomponents, relative to crucial variables such as age and IQ, is similar to development within typically developing 
HOT \& COOL EF TRAJECTORIES IN ASD

groups. Measures of both hot (i.e. delay discounting, affective decision making) and cool EF (i.e. inhibition, working memory, planning) were the outcome variables. This may shed more light on the similarities and differences between hot and cool EF developmental pathways followed in ASD relative to neurotypical controls as well as provide a solid ground for longitudinal examinations of these trajectories.

A secondary aim was to investigate the association and internal organisation of hot and cool EF in ASD. Based on evidence from typical development (Hooper et al., 2004; Prencipe et al., 2011), it was hypothesised that cool and hot EF skills would be (even weakly) associated in the control group.We sought to determine whether the ASD group would present a similar pattern.

\section{Method}

\section{Participants}

Seventy nine children and adolescents (79) with an official diagnosis of ASD ( $M=11.27$ years, $S D=2.56$ ) (65 males) and ninety one (91) controls ( $M=10.80$ years, $S D=2.49)$ (60 males) aged 7-16 years old who were drawn from a larger longitudinal study were recruited from thirty mainstream and special education schools to participate in the present study. All ASD participants were high functioning, held an official clinical diagnosis by a qualified clinician using DSM-IV criteria (American Psychiatric Association, 1994) and qualified for a "broad ASD” on the Autism Diagnostic Interview/Autism Diagnostic Interview-Revised (ADI/ADIR; Le Couteur et al., 1989; Lord, Rutter, \& Le Couteur, 1994”) and/or the Autism Diagnostic Observation Schedule (ADOS; Lord et al., 2000), in accordance to National Institute for Health and Clinical Excellence (NICE, 2011) guidelines. They were also in receipt of a Statement of Special Educational Needs (SEN), a legal document that details the child's needs and services that the local authority has a duty to provide, which specified ASD as their primary need. All 
HOT \& COOL EF TRAJECTORIES IN ASD

clinical records were inspected and any individual lacking detailed information about the official source of diagnosis was excluded from the study. Additional exclusion criteria for the ASD group included the presence of a diagnosed psychiatric illness, comorbid conditions (i.e. ADHD, seizures or colour blindness) and Full Scale Intelligence Quotient (FSIQ) below 70 as determined by the abbreviated version of the Wechsler Intelligence scales (two subtests: vocabulary and matrix reasoning; Wechsler, 1999). Ninety one (91) typically developing children and adolescents were recruited from mainstream primary $(n=56$; Year 2-Year 6) and secondary schools ( $\mathrm{n}=35$; Year 7-Year 11$)$. Typically developing participants were required to have no diagnosis, and no family history of ASD, other mental health disorders, ADHD, dyslexia or learning disability. Participants were matched for chronological age $(t(170)=-$ $1.21, p=.23)$ and IQ $(t(170)=1.79, p=.08)$. Ethical approval for the study was obtained and all participants' parents/carers gave written informed consent. Table 1 shows descriptive characteristics (means and standard deviations) of participants of both groups. [Table 1 should be placed here]

\section{Measures}

\section{Cool Executive Function Tasks}

Inhibition. The 'R' and 'P' version of the Go/No-Go paradigm (Mueller \& Piper, 2014) was used to assess participants' response inhibition. An image of either the letter P or letter R appeared in the centre of the screen (for 1500 milliseconds) on a black background. Participants were told to press the button only when the letter P was shown (Go trials) and to avoid pressing it for the letter R (No-Go trials). The go / no-go ratio was 4:1 (80\% go trials, $20 \%$ no-go trials). On the second block of trials, the pattern was reversed and the participants were asked to press the button when the letter R appeared (Go trials) and to avoid pressing it when $\mathrm{P}$ was presented 
HOT \& COOL EF TRAJECTORIES IN ASD

(No-Go trials) this time. Participants were not provided with feedback after a correct or incorrect response. On this block, the go / no-go ratio was 1:4 (20\% go trials, $80 \%$ no-go trials). Before each block, participants first completed 10 practice trials followed by the actual 320 test trials. Participant response inhibition was noted, by recording the proportion of errors (incorrect No-Go trials). Lower scores indicated better performance.

Planning. Planning ability was assessed by the Tower of London (ToL) task (Shallice, 1982). Participants were presented with three 3 -move problems as a practice, followed by the 12 actual trials of the original problem set (two 2-move tasks; two 3-move tasks; four 4-move tasks; and four 5-move tasks). Successful performance required participants to solve each problem moving only one bead each time and in the number of moves required. Following the procedure of Monks et al. (2005) participants were given two minutes to complete each problem. The task was ceased when the participants completed all problems or failed two of them consecutively. In terms of scoring, the number of problems each participant completed successfully was recorded. One point was given to participants if they completed the problem successfully and 0 points if they failed to complete the problem. Scores ranged from 0 to 12 .

Working Memory. The digit span forward and backwards subtests from the Wechsler Intelligence Scale for Children-3rd edition were used to measure participants' verbal working memory (WISC-III; Wechsler, 1991). Participants were asked to repeat the sequence presented by the researcher (at a rate of one number per second) in the exact same order. In the backwards digit recall task, participants were asked to repeat the series of numbers in reverse order. If participants responded successfully to all trials (4) within a block, the researcher proceeded to the next block. At each span length each block included 2 trials. In terms of scoring, participants were awarded 1 point for each correct trial and the task was terminated when the participant failed both trials at any given span length. The sum of the points awarded for both the forward and backward subtest created a composite working memory score. 
HOT \& COOL EF TRAJECTORIES IN ASD

\section{Hot Executive Function Tasks}

Affective Decision Making. A modified computerised version of the IOWA gambling task (IGT; Bechara et al., 1994) was used to measure participants' affective decision making skills. Participants were presented with four decks of cards (A, B, C, and D) and were asked to pick a card from any of the four decks each time. Decks A and B were equivalent in terms of overall net loss, whereas decks $C$ and $D$ were equivalent in terms of overall net winning. For each card selection, the wins and losses were set in a way that in every block of 20 cards from Decks A or B there was a potential total gain of $£ 1,000$, interrupted by potential losses up to $£ 1,250$. Losses were less frequent but of a larger magnitude in deck B whereas in Deck A losses were more frequent but in smaller amounts. For Decks C and D, the wins for each block were $£ 500$ totally while the potential net losses $£ 250$. In Deck D losses were less frequent and of higher magnitude relative to those in Deck C. Thus, Decks A and B were equally "disadvantageous" relative to Decks C and D that were equally “advantageous". Following Verdejo-Garcia et al. (2006), scores were calculated by subtracting the number of disadvantageous choices (decks A and B) from the number of advantageous choices (decks C and D) divided then by the total number of trials.

Delay Discounting. In line with previous research studying hot EF (Hongwanishkul et al., 2005; Prencipe et al., 2011), the Delay Discounting task was used in the present study in a computerised version to assess participants' ability to discount rewards (Richards et al., 1999). This task originally included the forced-choice between different amounts of money after different delays or with different chances as well. However, as the task was being given to a wide age range, including school aged children, it was modified to remove the probability questions. Participants were told that they had to select (hypothetically) between an immediate amount of money or $£ 10$ available after a delay. The test consisted of about 70 such questions 
HOT \& COOL EF TRAJECTORIES IN ASD

(i.e. (a) Would you rather have $£ 10$ for sure in 30 days or (b) $£ 2$ for sure right now?). The amount of immediate money was adjusted until the participant was indifferent between the two choices (random adjusting procedure; Richards et al., 1999). For every participant, this indifference point signified the effective value of the delayed large reward relative to an immediate amount of money (Richards et al., 1999). Delay discounting was determined by five delays $(0,10,30,180$, and 365 days later). In terms of scoring we followed the same procedure as Myerson et al.'s (2001), where the indifference points were used to estimate delay discounting. Thus, indifference points were established within participants and were plotted against time (delay). Indifference points and delays were normalised, by expressing indifference points as proportions of the amount of the maximum delayed reward (£10) and the delays as proportions of the maximum delay (365 days). These normalised values were used as the $\mathrm{x}$ (delay) and $\mathrm{y}$ (indifference points) axes in order to plot the discounting function. Separate trapezoids were then created by drawing vertical lines from each data point on the $\mathrm{x}$ axis. The formula $\left(\mathrm{x}_{2}-\mathrm{x}_{1}\right) \cdot\left[\left(\mathrm{y}_{1}+\mathrm{y}_{2}\right) / 2\right]$ was used to calculate the area of each trapezoid. The areas under these discounting curves (AUC) were calculated by summing the resulting trapezoids. The area under the curve (AUC) can range from 1 (no discounting) to 0 (maximum discounting). Larger numbers thus represent less discounting by delay (less impulsivity/ more self-control).

\section{Statistical analysis}

Preliminary Analysis: Group differences were investigated by conducting ANOVAs for each hot and cool EF measure in order to assess the average group differences for EF measures. Pearson's correlations were also performed between all EF measures, chronological age and IQ. 
HOT \& COOL EF TRAJECTORIES IN ASD

Main Analysis: Developmental cross-sectional trajectories were assessed employing the methods outlined by Thomas et al. (2009) for both hot and cool EF measures relative to chronological age and IQ. This procedure is very similar to ANOVA but differentiates by evaluating the differences between the slope and intercept of the lines depicting the developmental trajectory of each group instead of comparing the cross-sectional group means. A principal advantage of this analysis approach is the estimate of differences between groups in the continuous relationship between the dependent measure and other theoretically chosen constructs, allowing these conclusions to be drawn. The main effect of group (ASD or control), main effects of predictors (chronological age, IQ) and the interactions between group and slope were investigated.

\section{Results}

\section{Preliminary analysis}

Before turning to the trajectory analyses, we assessed average group differences in each EF task to allow direct comparisons between our samples and the extant literature. Significant group differences were found between the two groups performances both on cool EF: Go/NoGo $\left(F(1,163)=23.08, p<.001, \eta^{2}=.13\right)$, Digit $\operatorname{Span}\left(F(1,169)=28.21, p<.001, \eta^{2}=.14\right)$, $\operatorname{ToL}\left(F(1,168)=19.73, p<.001, \eta^{2}=.11\right)$ and hot EF tasks: $\operatorname{IGT}(F(1,165)=8.01, p=.005$, $\left.\eta^{2}=.05\right)$ and delay discounting $\left(F(1,147)=6.98, p=.009, \eta^{2}=.05\right)$. The ASD group showed significantly poorer performance in each hot and cool EF task relative to the control group (see Table 1 for Means and SDs).

Results of the correlational analysis between hot and cool EF and developmental predictors (age and IQ) separately in both groups are included in table 2. [Put table 2 here]

\section{Cross sectional developmental trajectories: cool executive function}


HOT \& COOL EF TRAJECTORIES IN ASD

Verbal working memory ability was assessed relative to chronological age using the digit span scores. The intercept of the trajectory was evaluated at the lowest age of overlap between the two groups (i.e. 7 years of age; 84 months) as well as the within-group trajectory slopes. The intercepts of digit span trajectory were not significantly different between the two groups, $F(1,169)=.25, p=.62$, partial $\eta^{2}=.001$ suggesting that at the lowest age of overlap, performance was similar for both groups (no delayed onset of development). In terms of rate of change across age, chronological age was a significant predictor of the digit span scores, $F$ $(1,169)=25.39, p<.001$, partial $\eta^{2}=.13$. Moreover, it was found that there was a significant Group x Chronological Age interaction, $F(1,169)=11.28, p=.001$, partial $\eta^{2}=.06$. As indicated in table 3 and figure 1, for the control group, digit span scores improved with age while for the ASD group there were no significant age-related improvements.

The developmental trajectory of digit span was also evaluated against IQ in terms both of intercept at the lowest point of overlap between the groups (Wechsler's scale score of 70-the lowest score reported) and within-group slopes. The intercept of the digit span trajectory differed significantly between the two groups $F(1,169)=4.63, p=.03$, partial $\eta^{2}=.03$, indicating that at the lowest point for IQ there was an initial difference between the two groups on digital performance. IQ significantly predicted digit span scores $F(1,169)=6.53, p=.01$, partial $\eta^{2}=.04$; but this relationship was not statistically different between the two groups (no significant IQ x group interaction found) $F(1,169)=.03, p=.86$, partial $\eta^{2}<.01$. As seen in table 3 and figure 1, digit span scores improved when IQ scores were higher, for both groups. [Put Figure 1 here]

Planning ability was assessed relative to chronological age using the ToL scores. The intercept of the trajectory was evaluated both at the lowest age of overlap between the two groups (i.e 84 months) and within-group trajectory slopes. The intercepts of the two groups did not significantly differ, $F(1,168)=.29, p=.594$, partial $\eta^{2}=.002$, suggesting that at the lowest 
HOT \& COOL EF TRAJECTORIES IN ASD

overlap between the two groups (84 months) performance was similar for the two groups (no delayed onset). However, in terms of rate of change, chronological age significantly predicted the ToL scores $F(1,168)=8.46, p=.004$, partial $\eta^{2}=.05$. A significant Group x Chronological Age interaction was found as well $F(1,168)=5.83, p=.017$, partial $\eta^{2}=.034$. As seen in table 3 and figure 2, for the control group there was a significant developmental trend for ToL scores to improve with chronological age, but for the ASD group there was no significant age-related difference across children and adolescents.

The developmental trajectory of ToL scores was also evaluated against IQ in terms of intercept both at the lowest point of overlap between the groups (Wechsler's scale score of 70-the lowest score reported) and within-group slopes. The intercept of ToL trajectory differed significantly between the two groups $F(1,168)=11.46, p=.001$, partial $\eta^{2}=.07$ at the lowest point of overlap between the two groups. IQ significantly predicted ToL scores, $F(1,168)=8.01, p=$ .005 , partial $\eta^{2}=.05$, but the IQ $x$ group interaction was not found significantly different, $F(1$, 168) $=2.79, p=.096$, partial $\eta^{2}=.02$. As seen in table 3 and figure 2 , for both groups, ToL scores improved when IQ scores were higher. [Put Figure 2 here]

Inhibition was assessed relative to chronological age using the go/no-go scores. The intercept of the trajectory was examined at the lowest age of overlap between the two groups (i.e. 84 months) as well as within-group trajectory slopes. Results showed that the intercept of the control group was significantly lower $F(1,163)=8.14, p=.005$, partial $\eta^{2}=.048$, indicating that performance of ASD participants was poorer at the lowest overlap between the two groups (delayed onset for the ASD group). For rate of change over development, chronological age was a significant predictor of the go/no-go scores $F(1,163)=7.36, p=.007$, partial $\eta^{2}=.044$. The Group X Chronological Age interaction was not significant $F(1,163)=.038, p=.85$, partial $\eta^{2}<.001$. Figure 3 shows that for both groups, there was a trend for performance on go/no go measure to improve with chronological age. 
HOT \& COOL EF TRAJECTORIES IN ASD

The evaluation of the Go/No Go trajectory against IQ in terms of the intercept took place at the lowest point of overlap between the groups (Wechsler's scale score of 70-the lowest score reported) as well as within-group slopes. In terms of groups intercepts we found no significant differences $F(1,163)=2.65, p=.11$, partial $\eta^{2}=.016$. Furthermore, concerning the rate of change across IQ, this was not a significant predictor of performance over all participants $F(1$, $163)=.001, p=.97$, partial $\eta^{2}<.01$ and no reliable interaction of Group x IQ, $F(1,163)=.42$, $p=.52$, partial $\eta^{2}=.003$ was found. As seen in figure 3 the two groups' trajectories are almost parallel indicating no reliable IQ-related changes. [Put Figure 3 here]

\section{Cross sectional developmental trajectories: hot executive function}

Affective decision making ability was assessed relative to chronological age using the Iowa Gambling Task scores. The intercept of the trajectory was evaluated at the lowest age of overlap between the two groups (i.e. 84 months) as well as within-group trajectory slopes. The intercept of IGT trajectory did not differ significantly between the two groups $F(1,165)=1.4, p=.24$, partial $\eta^{2}=.009$, indicating no delayed onset in the ASD group relative to the control group. Chronological age was not a significant predictor of IGT scores $F(1,165)=.87, p=.35$, partial $\eta^{2}=.005$. Finally, there was no significant Group x Age interaction effect, $F(1,165)=.17, p$ $=.68$, partial $\eta^{2}=.001$. As shown in figure 4 for both groups, trajectories are almost parallel and performance did not present significant changes across younger and older participants.

The evaluation of the IGT trajectory against IQ in terms of the intercept took place at the lowest point of overlap between the groups (Wechsler's scale score of 70-the lowest score reported) as well as within-group slopes. There were no significant group differences $F(1,165)=.39, p$ $=.53$, partial $\eta^{2}=.002$ at the lowest point of overlap. Rate of change across IQ was not a significant predictor of performance, $F(1,165)=1.73, p=.19$, partial $\eta^{2}=.01$, nor was there 
HOT \& COOL EF TRAJECTORIES IN ASD

a significant interaction of Group x IQ, $F(1,165)=.44, p=.51$, partial $\eta^{2}=.003$. For both groups there were no significant IQ-related changes (see figure 4). [Put Figure 4 here]

Delay discounting was assessed relative to chronological age using the delay discounting scores. The intercept of the trajectory was examined at the lowest age of overlap between the two groups (i.e. 84 months) as well as within-group trajectory slopes. The intercept of delay discounting trajectory differed significantly between the two groups, $F(1,147)=5.19, p=$ .024 , partial $\eta^{2}=.04$. Chronological age was not a significant predictor of the delay discounting scores $F(1,147)=.26, p=.61$, partial $\eta^{2}=.002$, and there was no significant Group x Age interaction effect, $F(1,147)=1.10, p=.29$, partial $\eta^{2}=.008$. As shown in figure 5 , for both groups, performance did not present significant changes across younger and older participants. The evaluation of the delay discounting trajectory against IQ in terms of the intercept took place at the lowest point of overlap between the groups (Wechsler's scale score of 70-the lowest score reported) as well as within-group slopes. There were no significant group differences $F$ $(1,147)=.47, p=.49$, partial $\eta^{2}=.003$ at the lowest point of overlap. For rate of change over IQ results showed that IQ was not a significant predictor of performance over all participants $F$ $(1,147)=.15, p=.7$, partial $\eta^{2}=.001$, and no reliable interaction of Group x IQ, $F(1,147)=$ $.37, p=.54$, partial $\eta^{2}=.003$ was found. For both groups there were no significant IQ- related changes (figure 5). [Put Figure 5 here] [Put Table 3 here]

\section{Associations between hot and cool tasks}

Correlational analyses performed separately in the two groups (see table 2) showed that cool and hot EF measures were significantly correlated only in the control group. More specifically, IGT scores were correlated to Digit Span and ToL scores. No significant associations were found between hot and cool EF in the ASD group. 
HOT \& COOL EF TRAJECTORIES IN ASD

\section{Discussion}

The purpose of the present study was to characterize profiles of hot and cool EF development in school-aged children and adolescents with ASD using directly assessed typically developing children as a point of reference. To date, research on EF development in ASD has focused on cool EF aspects, failing to integrate the hot EF processes. Thus, the current study sought to extend the investigation of EF development between children and adolescents with and without ASD by employing a more extensive battery of both cool and hot EF tasks in comparison to previous studies (Geurts et al., 2014). In contrast to testing differences in cross-sectional group means, which masks changes associated with age or mental ability (IQ), the cross-sectional developmental trajectories approach was adopted to avoid the methodological limitations of previous studies on this topic. Only cool EF, inhibition presented age-related improvements in the ASD sample while planning and working memory lacked significant developmental gains. In terms of IQ, again only cool EF, working memory and planning, presented changes between lower and higher IQ functioning participants in ASD. No age or IQ-related differences were found for hot EF aspects in either group. Our results extend evidence for intact and impaired aspects of developmental progression of distinct EF across childhood and adolescence in ASD.

\section{Cool and hot EF developmental trajectories}

Working memory is suggested to continue to improve throughout childhood and well into adolescence (i.e. Best \& Miller, 2010) as demonstrated by tasks tapping both visual and verbal working memory (Gathercole et al., 2004; Luciana \& Nelson, 2002). Our results indeed documented a developmental pattern of increases and a linear age trend for the typically developing participants that differed however to the developmental trajectory of the ASD 
HOT \& COOL EF TRAJECTORIES IN ASD

group. Even though at the age of onset ( 7 years of age) there were no differences between the two groups, the ASD group exhibited a developmental ceiling and never reached the performance level of controls in adolescence. This lack of significant age-related improvements is in line with recent reports of developmental arrest in verbal working memory in ASD (Andersen et al., 2014; Van den Bergh et al., 2014), while limited research has demonstrated that working memory impairments might increase with age within ASD likely due to a higher load for manipulation of working memory information during adolescence (Travers et al., 2011; Rosenthal et al., 2013). Our data could not reveal whether these working memory deficits are present across adulthood or whether maturation occurs in later adolescence/early adulthood, as we only included participants between 7-16 years. This pattern of deviant development suggests that working memory may be intact in preschool period but reaches a performance ceiling during middle childhood with deficits persisting in adolescence in ASD. Luna et al. (2007) suggests that if deficient performance is present later in development, as in the present case (early adolescence), that could imply that the developmental transition and underlying brain maturation mechanisms regulating verbal working memory (e.g. dorsolateral prefrontal cortex/ left temporo-frontal cortex) might be deficient in ASD. Unlike the differences in the age-related changes, both groups' performance was found to present improvements with higher IQ scores. The robust association between intelligence and working memory is well established in the literature, as high IQ scores are linked to more robust working memory capacity (Alloway et al., 2009). Working memory is argued to represent the "dynamic tradeoff" between the processing and storage of information, required in complex measures of intelligence and high-order cognition in general (Unsworth et al., 2014).

With regards to planning, results of the control group were consistent with previous reports of significant developmental changes throughout middle childhood and adolescence (Huizinga et 
HOT \& COOL EF TRAJECTORIES IN ASD

al., 2006; Korkman et al., 2001). Despite the lack of deficits at the age of onset (7 years), we found no significant age-related improvements across development in ASD. Our results reveal a deviant developmental pattern and support the limited number of previous studies reporting no age related improvements in planning in ASD (Ozonoff \& McEvoy, 1994; Ozonoff et al., 2004; Van den Bergh et al., 2014). Planning deficits may emerge in school age, especially across the developmental transition from primary to secondary settings where demands of the environment are higher. These findings contradict Chen et al.'s recent study (2016) showing that planning deficits in ASD as measured by Cantab's Stockings of Cambridge (SOC) were significant in childhood but would lessen with age. One possible explanation could be their participants exhibiting superior IQ scores (>10 points higher) relative to ours, while their significantly larger ASD sample size $(n=114)$ could have also allowed-in terms of statistical power- for the detection of subtle developmental changes. Similar to the working memory context, planning deficits seem to appear later in the development and pertain across age suggesting that the developmental transition from childhood to adolescence might be impaired for the underlying brain regions (i.e. dorsolateral prefrontal cortex) of planning skills (Luna et al., 2007) in ASD. Finally, the demonstration of planning scores improving with higher IQ scores is in line with previous studies reporting such significant association between intelligence and planning in ASD (Kimhi et al., 2014; Pellicano, 2007, van Eylen et al., 2015) and typical development (Arffa, 2007), as they are both core cognitive constructs contributing mutually towards the development of self-regulation.

Our results showed that inhibition improved with age but not with higher IQ scores in either group. The steady pattern of improvements from age 7 to 16 for controls corroborate reports of response inhibition tasks (i.e. Go/No-Go), following advances not only during early and middle childhood (Carlson et al., 2013; Carlson \& Moses, 2001; Romine \& Reynolds, 2005), 


\section{HOT \& COOL EF TRAJECTORIES IN ASD}

but beyond 10 years as well (even more subtle) (Best \& Miller, 2010). Most importantly, the significant age-related improvements reported in the ASD group, in line with previous studies (Happé et al., 2006a; Luna et al., 2007; van Eylen et al., 2015), paint a more positive picture of autistic children's particular cognitive developmental trends, indicating perhaps the likelihood of a window of plasticity in ASD as well. It should be noted however that the ASD group indicated a lower performance at the age of onset ( 7 years) which remained present throughout development in ASD without reaching the levels of neurotypicals. This evidence partially supports Happé et al.'s (2006b) theory proposing that there may be a particular profile of "coexisting cognitive atypicalities" in ASD that are present throughout development. Our data failed to provide more evidence about the maturity peak of inhibition in ASD (if they ever develop up to the same level as controls) as it did not include older adolescents or young adults. Looking at these significant improvements in inhibition from the maturation processes perspective of Luna et al. (2007), it could imply that impairments in the underlying brain mechanisms (i.e. dorsal areas of the lateral prefrontal cortex) are not related to the brain developmental/ maturation processes (that may be intact for ASD participants). The age-related improvements of inhibition in our ASD sample highlight the importance of implementing interventions aimed at augmenting self-control (inhibitory control) within ASD. The lack of significant developmental relationship between IQ and inhibition is in line with previous research investigating such association in school age in Attention-Deficit/Hyperactivity Disorder (ADHD) (Bitsakou et al., 2008; Rubia et al., 1998) and typical development (Lee et al., 2015) suggesting that intelligence does not explain any inhibition variance in ASD either.

To our knowledge, this was the first study to examine the development of hot EF in ASD across childhood and adolescence. Contrary to the findings for cool EF, hot EF demonstrated nonsignificant age related changes in both typical development and ASD. The lack of change in both groups' performance in hot EF across childhood and adolescence contradicted theories 
HOT \& COOL EF TRAJECTORIES IN ASD

from typical development suggesting that the development of hot EF measures would be protracted across childhood and early adolescence, following the extended development of the ventromedial prefrontal cortex (Segalowitz \& Davies, 2004). Indeed, selective studies in typical development addressing the Delay Discounting task (Scheres et al., 2006;) showed that younger participants (6-11 years) discounted rewards more steeply (lower performance) than older adolescents (12-17 years), while Hooper et al. (2004) reported that the oldest adolescents (14-17 years) performed better than younger participants (9-13 years) on the IGT task. However, Scheres et al. (2006) for example, used a temporal and probabilistic discounting task with a differentiated to ours design including smaller magnitudes of the immediate monetary reward $(0,2,4,6,8$, or 10 cents $)$, shorter delays $(0,5,10,20$, or 30 seconds) as well as probability levels of the large reward (more complex task) and a smaller number of trials. Moreover, the monetary awards used in their study were real rather than hypothetical and participants were paid a small amount of money after the practice trials (reinforcers). This could have perhaps made their older participants more motivated to wait for the larger rewards during task relative to participants in the current study. Moreover, in Hooper's et al. (2004) study, participants who exhibited a positive net earning at the end were actually paid that real amount of money as well, while in the present study monetary rewards were hypothetical due to the impractical cost and ethical issues raised within the school contexts. Generally, as constructs of delay discounting are complex and multifaceted, different aspects or task designs may follow different developmental pathways and such a more differentiated operationalisation is needed (Steinberg et al., 2009). Different hot EF measures seem to vary in motivational and emotional significance due to different designs or requirements; thus participants may vary in their performance or subtle developmental changes may get masked. As several measures of hot EF have been criticised for lacking enough "heat" or not being ecologically valid (Welsh \& Peterson 2014), one could argue that the hot EF tasks as used in this study (e.g. the Delay 
HOT \& COOL EF TRAJECTORIES IN ASD

Discounting was modified to lack the probability questions) were not so hot for the age group used here. For example, the differing quantities of imaginary money is quite an abstract construct that failed to enhance younger children's motivation or led the older ones to soon reach a performance ceiling.

In addition to this, Xu et al. (2016) very recently found that participants would demonstrate reduced risk taking in gambling tasks (tapping affective decision making) after money loss when the monetary awards were real relative to those receiving hypothetical rewards, suggesting amplified loss aversion (focus on avoiding losses rather than receiving gains) with real monetary awards. Thus, one possible explanation for our older participants not making choices that are more advantageous over the younger ones on the IGT could be that the hypothetical awards did not increase their sensitivity to money loss or enhance their desire to win. Our results are in line with neuroimaging evidence having shown that different age groups (8-10, 12-14, 16-17 years) did not differ in the recruitment of the dorsolateral prefrontal cortex during gambling tasks (van Leijenhorst et al., 2010) despite the dorsolateral prefrontal cortex being one of the last brain regions to reach maturity (Bunge \& Wright, 2007). Finally, as this is the first study to investigate the development of both hot and cool EF in ASD population, it could simply be the case of the hot EF trajectory not exhibiting any dramatic changes across middle childhood and adolescence. Our ASD sample included participants beyond early childhood (> 7 years) and we could assume that hot EF might present rapid changes during the preschool period in ASD. Future longitudinal studies are needed to clarify this issue.

\section{Cool and Hot EF organisation}

Following the different developmental trends found in the ASD group, different patterns of relations were also found among the cool and hot EF measures in the two groups. 
HOT \& COOL EF TRAJECTORIES IN ASD

Developmental theories that suggest the distinction between hot and cool EF (Zelazo \& Müller, 2002), argue that cool cognitive $\mathrm{EF}$ is regulated by lateral inferior and dorsolateral frontoparietal mechanisms (Miller \& Cummings, 2007) while hot affective EF is mainly mediated by the paralimbic orbitomedial and ventromedial frontolimbic structures (Fuster, 1997). Distinct neural regulations could allow for distinct developmental pathways; however very little is known about their organisation in ASD.

Interestingly, the association between hot and cool EF was significant only in the control group consistent with our hypothesis; affective decision making was significantly correlated to cool working memory and planning in line with previous studies investigating such a relationship in adulthood (Brand et al., 2005; Hinson et al., 2003). There is evidence though suggesting that performance in hot EF gambling tasks are independent of performance on cool EF measures, including working memory, in adults (Fonseca et al., 2012) and young childhood (O'Toole et al., 2016). Our results add to this ongoing debate in typical development by suggesting that affective decision making and cool EF are associated across middle childhood to adolescence. Overall, this evidence shows that during middle childhood and adolescence within typical development, hot and cool EF may not necessarily be considered as separate constructs (Allan \& Lonigan, 2014).

No correlations between hot and cool EF were found in ASD, suggesting that hot and cool EF could be dissociable functions in ASD. Generally, it is suggested that cool and hot EF aspects could differentiate from each other across development, extending early childhood (Diamond 2006). However, our results show that the internal EF organisation may represent a multidimensional model distinguishing between hot and cool only in the ASD group, but a unitary construct (Allan \& Lonigan, 2014) in typical development. More research using factor analysis in ASD is needed though, in order to clarify whether cool and hot EF are truly 


\section{HOT \& COOL EF TRAJECTORIES IN ASD}

representing distinct domains or a unitary construct, simply used differently under abstract and emotionally/motivationally significant situations in problem solving.

The findings of the present study need to be interpreted in the light of limitations. First, as we followed the convenience sampling approach, the relatively small number of children and adolescents that were recruited may not represent the broader ASD population. Although this study provided a more advanced insight into the development of the fractionated hot/cool EF model in ASD, it only included 7 to-16-year-old participants; it thus remains to be explored whether these findings can be attributed to younger children, or adults across the autism spectrum functioning levels. In addition, the present evidence needs to be interpreted cautiously as the statistical analysis used here (developmental trajectory approach) does not account for the correlation between age and IQ across the relationship of age to EF performance. Finally, the lack of a validated screening tool to support the provided clinical diagnostic reports of ASD was an important limitation.

In conclusion, the evidence from the present study suggests that shedding more light on the predictors and the developmental trajectories of cool and hot EF as well as their internal organisation, could contribute to our theoretical understanding of the brain maturation processes of children and adolescents with ASD. However, as this research is quite limited yet, findings should be interpreted cautiously. Future longitudinal studies are needed in order to corroborate between- group differences in the developmental trajectories of EF in ASD as found using cross-sectional approaches. The factors that contribute to hot/cool EF development and its organisation in ASD are complex and this cross-sectional study provides a basis for exploring these in future longitudinal work.

\section{Compliance with Ethical Standards:}

Funding: none. Ethical approval: All procedures performed in studies involving human participants were in accordance with the ethical standards of the institutional and/or national research committee and with 
HOT \& COOL EF TRAJECTORIES IN ASD

the 1964 Helsinki declaration and its later amendments or comparable ethical standards.

Informed consent: Informed consent was obtained from all individual participants included in the Study.

\section{References}

Albert, D., \& Steinberg, L. (2011). Age differences in strategic planning as indexed by the Tower of London. Child development, 82(5), 1501-1517.

Allan, N. P., \& Lonigan, C. J. (2014). Exploring dimensionality of effortful control using hot and cool tasks in a sample of preschool children. Journal of Experimental Child Psychology, 122, 33-47. doi: 10.1016/j.jecp.2013.11.013

Alloway, T. P., Gathercole, S. E., Kirkwood, H., \& Elliott, J. (2009). The cognitive and behavioral characteristics of children with low working memory. Child development, 80, 606-621. doi: 10.1111/j.1467-8624.2009.01282.x

Andersen, P. N., Skogli, E. W., Hovik, K. T., Geurts, H. M., Egeland, J., \& Oie, M. (2014). Working memory arrest in children with high-functioning autism compared to children with attention-deficit/hyperactivity disorder: Results from a 2-year longitudinal study. Autism. doi: https://doi.org/10.1177/1362361314524844

Anderson, V. (1998). Assessing executive functions in children : Biological, psychological, and developmental considerations. Neuropsychological Rehabilitation, 8,319-349.

Anderson, V. A., Anderson, P., Northam, E., Jacobs, R., \& Catroppa, C. (2001). Development of executive functions through late childhood and adolescence in an Australian sample. Developmental neuropsychology, 20(1), 385-406.

American Psychiatric Association (APA) (1994). Diagnostic and statistical manual of mental disorders (4th ed.). Washington, DC: Author.

Arffa, S. (2007). The relationship of intelligence to executive function and non-executive function measures in a sample of average, above average, and gifted youth. Archives of Clinical Neuropsychology, 22, 969-978. doi: 10.1016/j.acn.2007.08.001

Asato, M. R., Sweeney, J. A., Luna, B. Cognitive processes in the development of TOL performance. Neuropsychologia, 44, 2259-2269.

Bechara, A. (2004). The role of emotion in decision-making: Evidence from neurological patients with orbitofrontal damage. Brain and Cognition, 55, 30- 40. doi: 10.1016/j.bandc.2003.04.001

Bechara, A., Damasio, H., \& Damasio, A. R. (2000). Emotion, decision making and the orbitofrontal cortex. Cerebral Cortex, 10, 295-307.

Bechara, A., Damasio, A. R., Damasio, H., \& Anderson, S. W. (1994). Insensitivity to future consequences following damage to prefrontal cortex. Cognition, 50, 7-15.

Best, J. R., \& Miller, P. H. (2010). A developmental perspective on executive function. Child development, 81(6), 1641-1660.

Bitsakou, P., Psychogiou, L., Thompson, M., \& Sonuga-Barke, E. J. (2008). Inhibitory deficits in attention-deficit/hyperactivity disorder are independent of basic processing efficiency and IQ. Journal of Neural Transmission, 115, 261-268. doi: 10.1007/s00702-007-0828-z

Brand, M., Fujiwara, E., \& Markowitsch, H. J. (2005). Decision-making deficits of Korsakoff patients in a new gambling task with explicit rules: Associations with executive functions. Neuropsychology, 19, 267-277. doi: 10.1037/08944105.19.3.267

Bunge, S. A., \& Wright, S. B. (2007). Neurodevelopmental changes in working memory and cognitive control. Current Opinion in Neurobiology, 17, 243-250. doi: 10.1016/j.conb.2007.02.005

Carlson, S. M., \& Moses, L. J. (2001). Individual differences in inhibitory control and children's theory of mind. Child Development, 72, 1032-1053. doi: 10.1111/1467-8624.00333 
HOT \& COOL EF TRAJECTORIES IN ASD

Carlson, S. M., \& Zelazo, P. D., \& Faja, S. (2013). Executive function. In P. D. Zelazo (Ed.), The Oxford handbook of developmental psychology, Vol. 1: Body and mind (pp. 706-743). New York: Oxford University Press.

Carper, R. A., \& Courchesne, E. (2005). Localized enlargement of the frontal cortex in early autism. Biological psychiatry, 57(2), 126-133.

Chen, S. F., Chien, Y. L., Wu, C. T., Shang, C. Y., Wu, Y. Y., \& Gau, S. S. (2016). Deficits in executive functions among youths with autism spectrum disorders: an age-stratified analysis. Psychological Medicine, 46, 1625-1638. doi: $10.1017 / \mathrm{S} 0033291715002238$

De Luca, C. R., Wood, S. J., Anderson, V., Buchanan, J. A., Proffitt, T. M., Mahony, K., \& Pantelis, C. (2003). Normative data from the CANTAB. I: development of executive function over the lifespan. Journal of Clinical and Experimental Neuropsychology, 25, 242-254. doi: 10.1076/jcen.25.2.242.13639

Diamond, A. (2006). The early development of executive functions. In E. Bialystok \& F. I. M. Craik (Eds.), Lifespan cognition: Mechanisms of change (pp. 70-95). New York, NY: Oxford University Press.

Duncan, J. \& Owen, A. M. (2000). Common regions of the human frontal lobe recruited by diverse cognitive demands. Trends Neuroscience. 23, 475-483.

Fonseca, R. P., Zimmermann, N., Cotrena, C., Cardoso, C., Kristensen, C. H., Grassi-Oliveira, R. (2012). Neuropsychological assessment of executive functions in traumatic brain injury: hot and cold components. Psychological Neuroscience, 5, 183-190. doi: http://dx.doi.org/10.3922/j.psns.2012.2.08

Funahashi, S. (2004). Information processing in the primate prefrontal cortex. In S. Otani (Ed.), Prefrontal cortex: From synaptic plasticity to cognition. Boston, MA: Kluwer Academic Publishers.

Fuster, J. M. (1997). The Prefrontal Cortex. New York: Raven Press.

Garon, N., Bryson, S. E., Smith, I. M. (2008). Executive function in preschoolers: A review using an integrative framework. Psychological Bulletin, 134, 31-60.

Gathercole, S. E., Pickering, S. J., Ambridge, B., Wearing, H. (2004). The structure of working memory from 4 to 15 years of age. Developmental Psychology, 40, 177-190.

Geurts, H. M., de Vries, M. \& van den Bergh, S. F. (2014). Executive functioning theory and autism. In: S. Goldstein \& J. Naglier (Eds), Handbook of executive functioning (pp.121-141). New York: Springer.

Gilbert, S. J., \& Burgess, P. W. (2008). Executive function. Current Biology, 18(3), R110-114.

Griebling, J., Minshew, N. J., Bodner, K., Libove, R., Bansal, R., Konasale, P., ... \& Hardan, A. (2010). Dorsolateral prefrontal cortex magnetic resonance imaging measurements and cognitive performance in autism. Journal of child neurology, 25(7), 856-863.

Happé, F., Booth, R., Charlton, R., \& Hughes, C. (2006a). Executive function deficits in autism spectrum disorders and attention-deficit/hyperactivity disorder: examining profiles across domains and ages. Brain and cognition, 61, 25-39. doi: 10.1016/j.bandc.2006.03.004

Happé, F., Ronald, A., \& Plomin, R. (2006b). Time to give up on a single explanation of autism. Nature Neuroscience, 9 , 1218-1220.

Hill, E. L. (2004). Evaluating the theory of executive dysfunction of autism. Developmental Review, 24, 189-233. doi: 10.1016/j.dr.2004.01.001

Hill, E. L., \& Frith, U. (2003). Understanding autism: insights from mind and brain. Philosophical Transactions of the Royal Society of London B: Biological Sciences, 358(1430), 281-289.

Hinson, J. M., Jameson, T. L., \& Whitney, P. (2003). Impulsive decision making and working memory. Journal of Experimental Psychology: Learning, Memory, and Cognition, 29, 298-306. doi: http://dx.doi.org/10.1037/02787393.29.2.298 
HOT \& COOL EF TRAJECTORIES IN ASD

Hongwanishkul, D., Happaney, K. R., Lee, W. S, \& Zelazo, P. D. (2005). Assessment of hot and cool executive function in young children: Age-related changes and individual differences. Developmental Neuropsychology, 28. doi: $10.1207 / \mathrm{s} 15326942 \mathrm{dn} 2802 \_4$

Hooper, C. J., Luciana, M., Conklin, H. M., \& Yarger, R. S. (2004). Adolescents' performance on the Iowa Gambling Task: Implications for the development of decision-making and ventromedial prefrontal cortex. Developmental Psychology, 40, 1148-1158. doi: 10.1037/0012-1649.40.6.1148

Huizinga, M., Dolan, C. V., \& van der Molen, M. W. (2006). Age-related change in executive function: Developmental trends and a latent variable analysis. Neuropsychologia, 44, 2017-2036

Johnstone, S. J., Dimoska, A., Smith, J. L., Barry, R. J., Pleffer, C. B., Chiswick, D., et al. (2007). The development of stopsignal and go/nogo response inhibition in children aged 7-12 years: Performance and event related potential indices. International Journal of Psychophysiology, 63, 25-38.

Just, M. A., Cherkassky, V. L., Keller, T. A., \& Minshew, N. J. (2004). Cortical activation and synchronization during sentence comprehension in high-functioning autism: evidence of underconnectivity. Brain, 127(8), 1811-1821.

Kim, S., Nordling, J. K., Yoon, J. E., Boldt, L. J., \& Kochanska, G. (2014). Effortful control in "hot" and "cool” tasks differentially predicts children's behaviour problems and academic performance. Journal of Abnormal Child Psychology, 41, 43-56. doi: 10.1007/s10802-012-9661-4

Kimhi, Y., Kugelmas, D., Agam Ben Artzi, G., Ben Moshe, I., \& Bauminger-Zviely, N. (2014). Theory of mind and executive function in preschoolers with typical development versus intellectually able pre-schoolers with autism spectrum disorder. Journal of Autism and Developmental Disorders, 44. doi: 10.1007/s10803-014-2104-z

Konishi, S., Jimura, K., Asari, T., \& Miyashita, Y. (2003). Transient activation of superior prefrontal cortex during inhibition of cognitive set. Journal of Neuroscience, 23, 7776-7782.

Korkman, M., Kemp, S. L., \& Kirk, U. (2001). Effects of age on neurocognitive measures of children ages 5 to 12: A crosssectional study on 800 children from the United States. Developmental neuropsychology, 20(1), 331-354.

Koshino, H., Carpenter, P. A., Minshew, N. J., Cherkassky, V. L., Keller, T. A., \& Just, M. A. (2005). Functional connectivity in an fMRI working memory task in high-functioning autism. Neuroimage, 24(3), 810-821.

Krain, A. L., Wilson, A. M., Arbuckle, R., Castellanos, F. X., Milham, M. P. (2006). Distinct neural mechanisms of risk and ambiguity: A meta-analysis of decision-making. NeuroImage, 32(1), 477-484.

http://dx.doi.org/10.1016/j.neuroimage.2006.02.047.

Le Couteur, A., Rutter, M., Lord, C., Rios, P., Robertson, S., Holdgrafer, M., \& McLennan, J. D. (1989). Autism Diagnostic Interview: A standardized investigator-based instrument. Journal of Autism and Developmental Disorders, 19, 363387.

Lee, H. W., Lo, Y. H., Li, K. H., Sung, W. S., \& Juan, C. H. (2015). The relationship between the development of response inhibition and intelligence in preschool children. Frontiers in Psychology, 6, 802. doi: https://doi.org/10.3389/fpsyg.2015.00802

Lord, C., Rutter, M., DiLavore, P. C., \& Risi, S. (2000). Autism Diagnostic Observation Schedule (ADOS). Los Angeles, California: Western Psychological Services.

Lord, C., Rutter, M., \& Le Couteur, A. (1994). Autism Diagnostic Interview-Revised: A revised version of a diagnostic interview for caregivers of individuals with possible pervasive developmental disorders. Journal of Autism and Developmental Disorders, 24, 659-685.

Luciana, M., \& Nelson, C. A. (2002). Assessment of neuropsychological function through use of the Cambridge Neuropsychological Testing Automated Battery: Performance in 4- to 12-year-old children. Developmental Neuropsychology, 22, 595-624. doi: 10.1207/S15326942DN2203_3 
HOT \& COOL EF TRAJECTORIES IN ASD

Luna, B., Doll, S. K., Hegedus, S. J., Minshew, N. J., \& Sweeney, J. A. (2007). Maturation of executive function in autism. Biological psychiatry, 61, 474-481. doi: 10.1016/j.biopsych.2006.02.030

McPartland, J., Dawson, G., Webb, S. J., Panagiotides, H., \& Carver, L. J. (2004). Event-related brain potentials reveal anomalies in temporal processing of faces in autism spectrum disorder. Journal of Child Psychology and Psychiatry, 45(7), 1235-1245.

Mak-Fan, K. M., Taylor, M. J., Roberts, W., \& Lerch, J. P. (2012). Measures of cortical grey matter structure and development in children with autism spectrum disorder. Journal of autism and developmental disorders, 42(3), 419-427.

Manes, F., Sahakian, B., Clark, L., Rogers, R., Antoun, N., Aitken, M., \& Robbins, T. (2002). Decision-making processes following damage to the prefrontal cortex. Brain, 125, 624-639.

Metcalf, J., \& Mischel, W. (1999). A hot/cool-system analysis of delay of gratification: Dynamics of willpower. Psychological Review, 106, 3-19.

Miller, B. L., \& Cummings, J. L. (Eds.). (2007). The human frontal lobes: Functions and disorders. Guilford press.

Monks, C. P., Smith, P. K., \& Swettenham, J. (2005). Psychological correlates of peer victimisation in preschool: Social cognitive skills, executive function and attachment profiles. Aggressive Behavior, 31, 571-588. doi: http://dx.doi.org/10.1002/ab.20099

Mueller, S. T., \& Piper, B. J. (2014). The psychology experiment building language (PEBL) and PEBL test battery. Journal of neuroscience methods, 222, 250-259. doi: 10.1016/j.jneumeth.2013.10.024

Myerson, J., Green, L., \& Warusawitharana, M. (2001). Area under the curve as a measure of discounting. Journal of the experimental analysis of behaviour, 76, 235-243. doi: 10.1901/jeab.2001.76-235

National Institute for Health and Clinical Excellence (2011). Autism: recognition, referral and diagnosis of children and young people on the autism spectrum. (Clinical guideline 128.)

Otero, T. M., \& Barker, L. A. (2014). The frontal lobes and executive functioning. In S. Goldstein \& J. A. Naglieri (Eds.), Handbook of Executive Functioning (pp. 29-45). New York, USA: Springer.

O’Toole, S., Monks, C. P., \& Tsermentseli, S. (2016). Development of Cool and Hot Executive Function and Theory of Mind across Early to Middle Childhood. Social Development, 1-13. doi: 10.1111/sode.12231

Ozonoff, S., Cook, I., Coon, H., Dawson, G., Joseph, R. M., Klin, A., et al. (2004). Performance on Cambridge Neuropsychological Test Automated Battery subtests sensitive to frontal lobe function in people with autistic disorder: evidence from the Collaborative Programs of Excellence in Autism network. Journal of autism and developmental disorders, 34, 139-150. doi:10.1023/B:JADD.0000022605.81989.cc

Ozonoff, S., \& McEvoy, R. E. (1994). A longitudinal study of executive function and theory of mind development in autism. Development and psychopathology, 6, 415-431. doi: https://doi.org/10.1017/S0954579400006027

Pellicano, E. (2007). Links between theory of mind and executive function in young children with autism: Clues to developmental primacy. Developmental Psychology, 43, 974-990. doi: 10.1037/0012-1649.43.4.974

Pellicano, E. (2010). Individual differences in executive function and central coherence predict developmental changes in theory of mind in autism. Developmental psychology, 46(2), 530-544.

Phan, K. L., Wager, T. D., Taylor, S. F., \& Liberzon, I. (2004). Functional neuroimaging studies of human emotions. CNS Spectrums, 9(4), 258-266.

Prencipe, A., Kesek, A., Cohen, J., Lamm, C., Lewis, M. D., \& Zelazo, P. D. (2011). Development of hot and cool executive function during the transition to adolescence. Journal of experimental child psychology, 108, 621-637. doi: 10.1016/j.jecp.2010.09.008 
HOT \& COOL EF TRAJECTORIES IN ASD

Richards, J. B., Zhang, L., Mitchell, S. H., \& Wit, H. (1999). Delay or probability discounting in a model of impulsive behavior: effect of alcohol. Journal of the experimental analysis of behaviour, 71, 121-143. doi: 10.1901/jeab.1999.71-121

Romine, C. B., Reynolds, C. R. (2005). A model of the development of frontal lobe function: Findings from a meta-analysis. Applied Neuropsychology, 12, 190-201.

Rosenthal, M., Wallace, G. L., Lawson, R., Wills, M. C., Dixon, E., Yerys, B. E., \& Kenworthy, L. (2013). Impairments in real-world executive function increase from childhood to adolescence in autism spectrum disorders. Neuropsychology, 27(1), 13.

Rubia, K., Oosterlaan, J., Sergeant, J. A., Brandeis, D., van Leeuwen, T. (1998). Inhibitory dysfunction in hyperactive boys. Behavioural Brain Research, 94, 25-32. doi: 10.1027//1016-9040.4.2.109

Segalowitz, S., \& Davies, P. L. (2004). Charting the maturation of the frontal lobe: An electrophysiological strategy. Brain and Cognition, 55, 116-133. doi: 10.1016/S0278-2626(03)00283-5

Shallice, T. (1982). Specific impairments of planning. Philosophical Transactions of the Royal Society of London, B 298, 199-209.

Scheres, A., Dijkstra, M., Ainslie, E., Balkan, J., Reynolds, B., Sonuga-Barke, E., et al. (2006). Temporal and probabilistic discounting of rewards in children and adolescents: Effects of age and ADHD symptoms. Neuropsychologia, 44, 2092 - 2103. doi: 10.1016/j.neuropsychologia.2005.10.012

Steinberg, L., Graham, S., O’Brien, L., Woolard, J., Cauffman, E., \& Banich, M. (2009). Age differences in future orientation and delay discounting. Child development, 80, 28-44. doi: 10.1111/j.1467-8624.2008.01244.x

Thomas, M. S., Annaz, D., Ansari, D., Scerif, G., Jarrold, C., \& Karmiloff-Smith, A. (2009). Using developmental trajectories to understand developmental disorders. Journal of speech, language, and hearing research, 52, 336-358. doi: 10.1044/1092-4388(2009/07-0144

Thomason, M. E., Race, E., Burrows, B., Whitfield-Gabrieli, S., Glover, G. H., \& Gabrieli, J. D. (2009). Development of spatial and verbal working memory capacity in the human brain. Journal of cognitive neuroscience, 21(2), 316-332.

Travers, B. G., Klinger, M. R., Klinger, L. G. (2011). Attention and working memory in ASD. In: D. Fein (Ed.), The Neuropsychology of Autism (pp. 161-184). New York: Oxford University Press.

Unsworth, N., Fukuda, K., Awh, E., \& Vogel, E. K. (2014). Working memory and fluid intelligence: Capacity, attention control, and secondary memory retrieval. Cognitive psychology, 71, 1-26. doi: 10.1016/j.cogpsych.2014.01.003

Unterrainer, J. M., Owen, A. M. (2006). Planning and problem solving: From neuropsychology to functional neuroimaging. Journal of Physiology Paris, 99, 308-317.

Van den Bergh, S. F., Scheeren, A. M., Begeer, S., Koot, H. M., \& Geurts, H. M. (2014). Age related differences of executive functioning problems in everyday life of children and adolescents in the autism spectrum. Journal of autism and developmental disorders, 44, 1959-1971. doi: 10.1007/s10803-014-2071-4

Van Eylen, L., Boets, B., Steyaert, J., Wagemans, J. \& Noens, I. (2015). Executive functioning in autism spectrum disorders: Influence of task and sample characteristics and relation to symptom severity. European Child and Adolescent Psychiatry, 24. doi: 10.1007/s00787-015-0689-1

Van Leijenhorst, L., Moor, B. G., de Macks, Z. A. O., Rombouts, S. A., Westenberg, P. M., Crone, E. A. (2010). Adolescent risky decision-making: neurocognitive development of reward and control regions. Neuroimage, 51, 345-355. doi: 10.1016/j.neuroimage.2010.02.038.

Verdejo-Garcia, A., Bechara, A., Recknor, E. C., Perez-Garcia, M. (2006). Decision-making and the Iowa Gambling Task: Ecological validity in individuals with substance dependence. Psychologica Belgica, 46, 55-78. doi: http://doi.org/10.5334/pb-46-1-2-55 


\section{HOT \& COOL EF TRAJECTORIES IN ASD}

Wagner, A. D., Maril, A., Bjork, R. A., \& Schacter, D. L. (2001). Prefrontal contributions to executive control: fMRI evidence for functional distinctions with lateral prefrontal cortex. NeuroImage, 14, 1337-1347. doi: 10.1006/nimg.2001.0936

Wechsler, D. (1991). Wechsler Intelligence Scale for Children-Third Edition. San Antonio TX: Psychological Corporation. Wechsler, D. (1999). The Wechsler abbreviated scale for intelligence. San Antonio TX: The Psychological Corporation.

Welsh, M., \& Peterson, E. (2014). Issues in the conceptualization and assessment of hot executive functions in childhood. Journal of the International Neuropsychological Society, 20(2), 152-156.

Willoughby, M., Kupersmidt, J., Voegler-Lee, M., \& Bryant, D. (2011). Contributions of hot and cool self-regulation to preschool disruptive behavior and academic achievement. Developmental neuropsychology, 36, 162-180. doi: 10.1080/87565641.2010.549980

Xu, S., Pan, Y., Wang, Y., Spaeth, A. M., Qu, Z., \& Rao, H. (2016). Real and hypothetical monetary rewards modulate risk taking in the brain. Scientific Reports, 6.

Zelazo, P. D., \& Carlson, S. M. (2012). Hot and cool executive function in childhood and adolescence: Development and plasticity. Child Development Perspectives, 6, 354-360. doi: 10.1111/j.1750-8606.2012.00246.x

Zelazo, P. D., Qu, L., \& Müller, U. (2005). Hot and cool aspects of executive function: Relations in early development. In W. Schneider, R. Schumann-Hengsteler, \& B. Sodian (Eds.), Young Children's Cognitive Development: Interrelationships Among Executive Functioning, Working Memory, Verbal Ability and Theory of Mind (pp. 71 - 95). Mahwah, New Jersey: Lawrence Erlbaum Associates Inc.

Zelazo, P. D., \& Müller, U. (2002). Executive function in typical and atypical development. In U. Goswami (Ed.), Handbook of Childhood Cognitive Development (pp. 445-470). Oxford: Blackwell Publishing Ltd. 
HOT \& COOL EF TRAJECTORIES IN ASD

Table 1a. Participants' characteristics.

Group

\begin{tabular}{|c|c|}
\hline ASD & Control \\
\hline$(n=79)$ & $(n=91)$ \\
\hline
\end{tabular}

Age (in years)

$M(S D)$

$11.27(2.56) \quad 10.80(2.49)$

Range

7-16

7-16

FSIQ

$M(S D)$

$95.85(15.09) \quad 99.78(13.54)$

Range

$70-127$

$72-135$

Digit Span

$M(S D)$

$11.33(3.09)$

$13.97(3.38)$

28.21

$(\mathrm{p}<.001)$

ToL

$M(S D)$

7.05 (2.03)

$8.31(1.65)$

19.73

$(\mathrm{p}<.001)$

\section{Go/No-Go}

$M(S D)$

$$
\begin{aligned}
& 48.86(15.98) \quad 35.91(18.11) \quad 23.08 \\
& (\mathrm{p}<.001)
\end{aligned}
$$

\section{IGT}

$M(S D)$

$$
-.04 \text { (.19) }
$$

$.05(.22)$

8.01

$(\mathrm{p}=.005)$

\section{Delay}

Discounting

$.33(.12)$

$.38(.12)$

6.98

$M(S D)$

$$
(\mathrm{p}=.009)
$$

Note. ToL= Tower of London task; IGT= lowa Gambling Task. All EF scores are the raw test scores. 
HOT \& COOL EF TRAJECTORIES IN ASD

Table 1b. Distribution of the sample across the two groups.

\begin{tabular}{lcl}
\hline & $\begin{array}{l}\text { Control } \\
(n=91)\end{array}$ & $\begin{array}{l}\text { ASD } \\
(n=79)\end{array}$ \\
\cline { 1 - 1 } Age cohorts (in years) & & \\
\cline { 1 - 2 } $7-9$ & 35 & 22 \\
$10-12$ & 32 & 34 \\
$13-16$ & 24 & 23 \\
\hline
\end{tabular}


HOT \& COOL EF TRAJECTORIES IN ASD

Table 2. Correlation matrix for separate groups (control subjects below the diagonal and ASD subjects above the diagonal).

Note. $* \mathrm{p}<.05, * * \mathrm{p}<.01$; ToL: Tower of London, IGT: Iowa Gambling Task. 
HOT \& COOL EF TRAJECTORIES IN ASD

Table 3. Intercept and slope of linear developmental trajectories predicting EF measures based on putative developmental predictors.

\begin{tabular}{|c|c|c|c|c|c|c|c|}
\hline IQ & & $-.51 * *$ & .21 & $.34 * *$ & -.05 & .07 & -.07 \\
\hline Age & $-.32 * *$ & & .13 & .04 & $-.25 *$ & .05 & -.05 \\
\hline DigitSpan & .19 & $.57 * *$ & & $.25^{*}$ & -.01 & .28 & .15 \\
\hline ToL & .11 & $.45 * *$ & $.45 * *$ & & -.01 & -.08 & .07 \\
\hline Go/No-Go & -.05 & -.18 & $-.23 *$ & -.09 & & -.02 & .04 \\
\hline IGT & .14 & .12 & $.26^{*}$ & $.27 *$ & -.18 & & .13 \\
\hline \multirow[t]{2}{*}{ Discounting } & -.14 & -.14 & -.14 & -.12 & .03 & -.06 & \\
\hline & $\underline{\sigma}$ & $\underset{\leftarrow}{\stackrel{8}{\alpha}}$ & 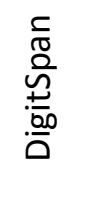 & $\vec{\circ}$ & $\begin{array}{l}\text { O } \\
\text { ’ } \\
\text { ż } \\
\text { ᄋ }\end{array}$ & 包 & 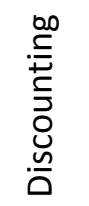 \\
\hline
\end{tabular}

ASD

Controls

\begin{tabular}{llll}
\hline Age & IQ & Age & IQ
\end{tabular}

Digit Span

\begin{tabular}{|c|c|c|c|}
\hline $\mathrm{m}=11.28 \quad(\mathrm{SE}=.34)$ & $\mathrm{m}=11.42(\mathrm{SE}=.37)$ & $\mathrm{m}=14.19(\mathrm{SE}=.31)$ & $\mathrm{m}=13.90 \quad(\mathrm{SE}=.34$ \\
\hline$b=9.56, r^{2}=.01$ & $b=7.22, r^{2}=.04$ & $b=5.65, r^{2}=.32$ & $r^{2}=.04$ \\
\hline
\end{tabular}

Go/No-Go

$$
\mathrm{m}=49.2(\mathrm{SE}=1.97) \quad \mathrm{m}=48.99(\mathrm{SE}=2.02)
$$

$\mathrm{m}=35.67 \quad(\mathrm{SE}=1.79)$

$\mathrm{m}=36.1 \quad(\mathrm{SE}=1.83)$

$\mathrm{b}=66.47, \mathrm{r}^{2}=.06$

$\mathrm{b}=44.14, \mathrm{r}^{2}=.002$

$\mathrm{b}=47.76, \mathrm{r}^{2}=.03$

$\mathrm{b}=42.00, \mathrm{r}^{2}=.002$

ToL

$\mathrm{m}=7.04 \quad(\mathrm{SE}=.20) \quad \mathrm{m}=7.15 \quad(\mathrm{SE}=.20)$

$\mathrm{m}=8.38 \quad(\mathrm{SE}=.19)$

$\mathrm{m}=8.29 \quad(\mathrm{SE}=.19)$

$b=6.74, r^{2}=.001 \quad b=2.75, r^{2}=.11$

$\mathrm{b}=5.09 \quad, \mathrm{r}^{2}=.2$

$\mathrm{b}=7.07, \mathrm{r}^{2}=.04$

IGT

$$
\begin{array}{lll}
\mathrm{m}=-.04 & (\mathrm{SE}=.024) & \mathrm{m}=-.036 \quad(\mathrm{SE}=.024) \\
\mathrm{b}=-.08 \quad, \mathrm{r}^{2}=.002 & \mathrm{~b}=-.12 \quad, \mathrm{r}^{2}=.005
\end{array}
$$

$\mathrm{m}=.06 \quad(\mathrm{SE}=.022)$

$\mathrm{m}=.05 \quad(\mathrm{SE}=.022)$

$\mathrm{b}=-.06, \mathrm{r}^{2}=.01$

$\mathrm{b}=-.18, \mathrm{r}^{2}=.01$

Delay Discount

$$
\begin{aligned}
& \mathrm{m}=.32 \quad(\mathrm{SE}=.015) \quad \mathrm{m}=.32 \quad(\mathrm{SE}=.015) \quad \mathrm{m}=.38 \quad(\mathrm{SE}=.014) \quad \mathrm{m}=.38 \quad(\mathrm{SE}=.014) \\
& \mathrm{b}=.3, \mathrm{r}^{2}=.002 \quad \mathrm{~b}=.38, \mathrm{r}^{2}=.006 \quad \mathrm{~b}=.45, \mathrm{r}^{2}=.01 \quad \mathrm{~b}=.37, \mathrm{r}^{2}<.001
\end{aligned}
$$

Note. ToL= Tower of London task; IGT= lowa Gambling Task. 
HOT \& COOL EF TRAJECTORIES IN ASD

Figure 1.
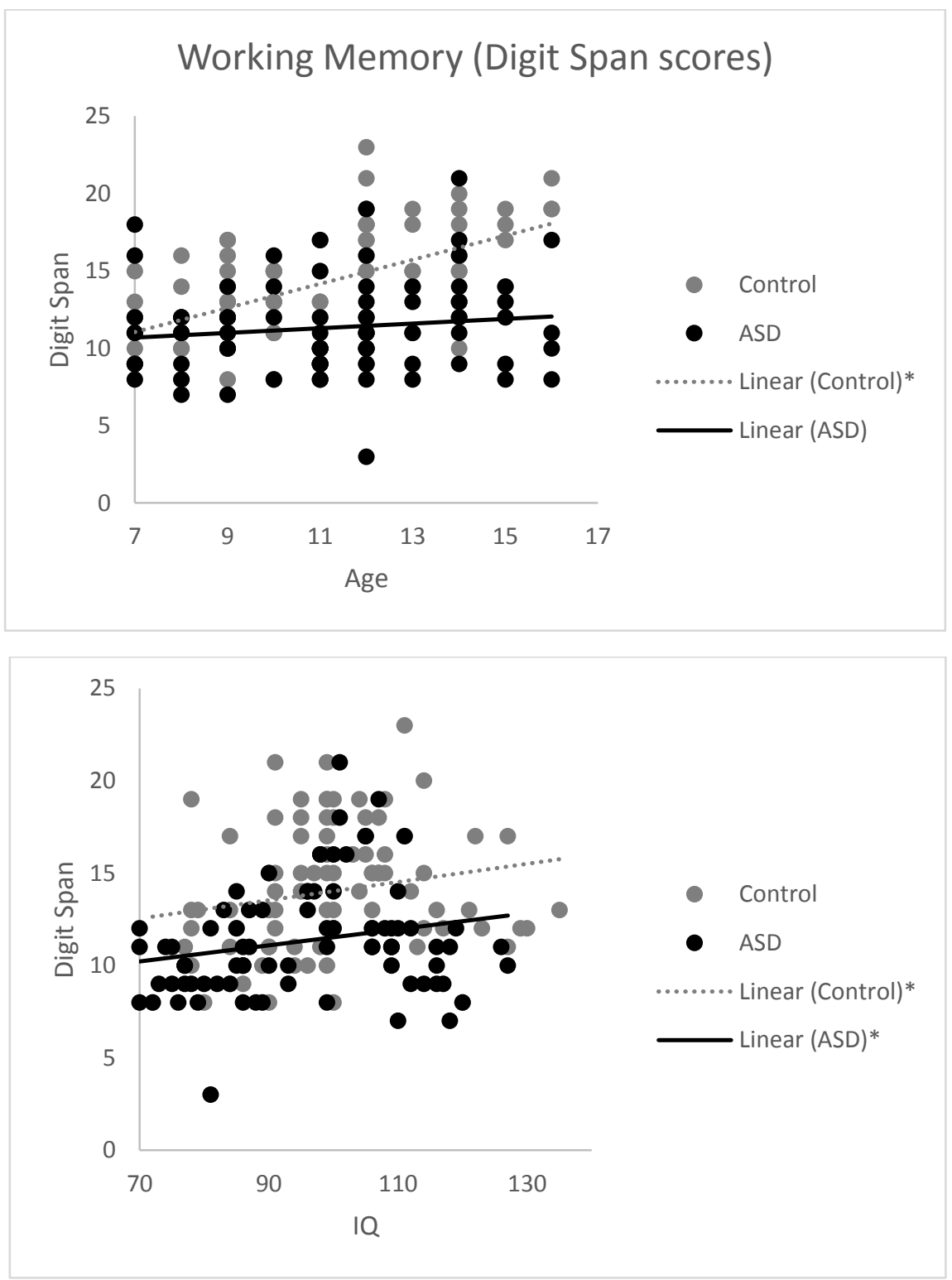
HOT \& COOL EF TRAJECTORIES IN ASD

$$
* p<.05
$$

Figure 2.

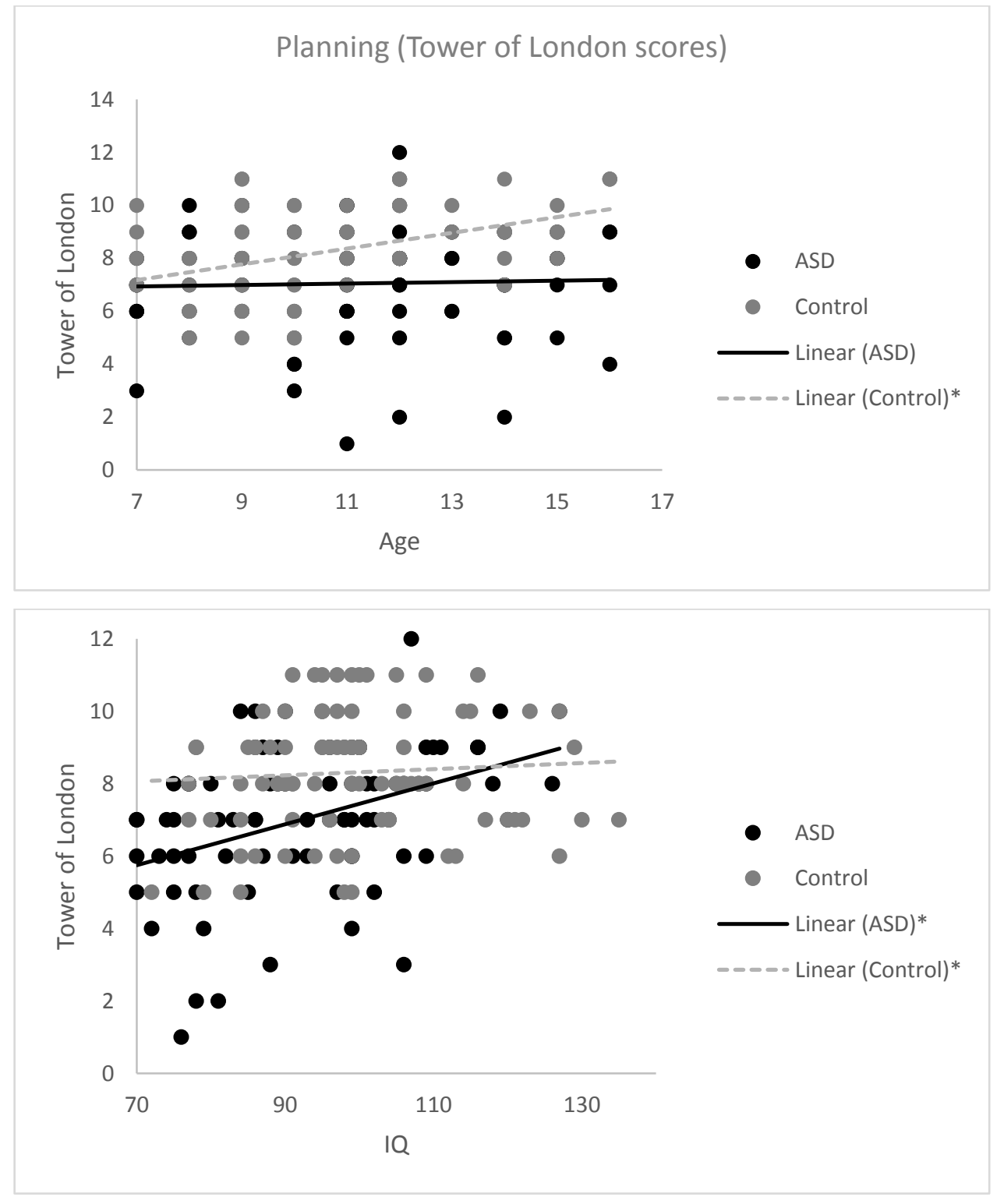


HOT \& COOL EF TRAJECTORIES IN ASD

$* P<.05$

Figure 3.

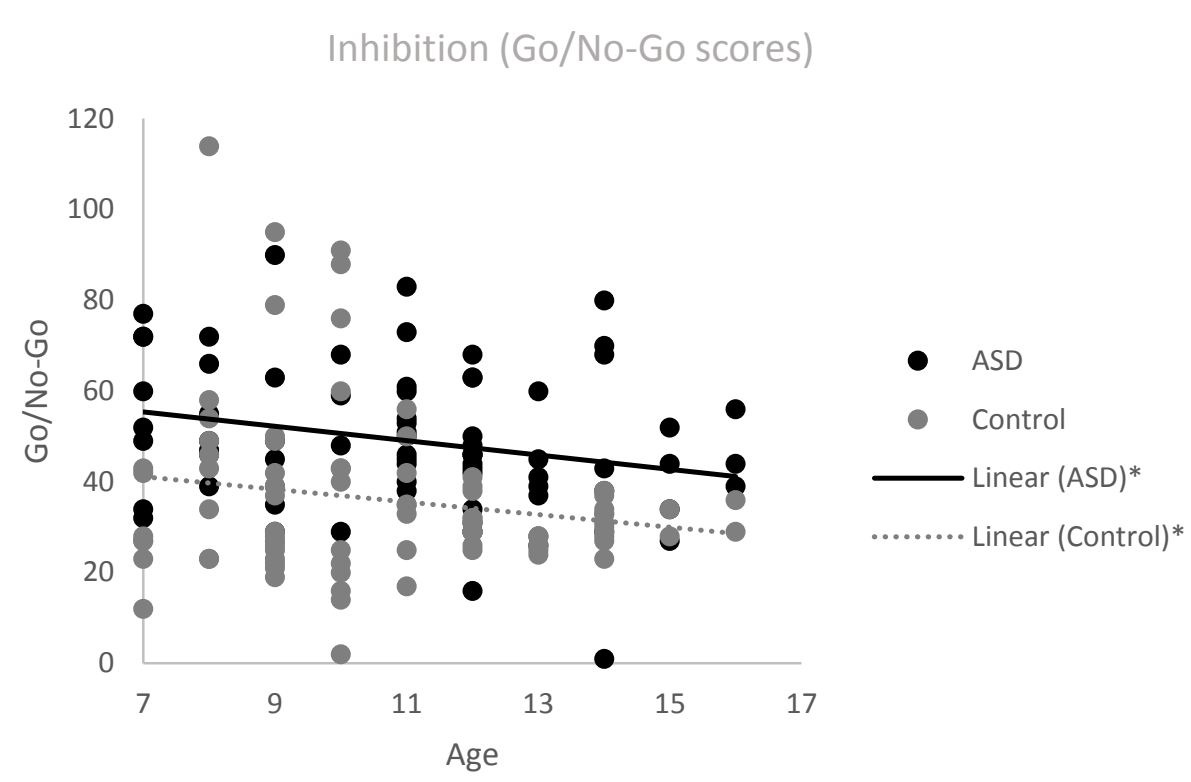


HOT \& COOL EF TRAJECTORIES IN ASD

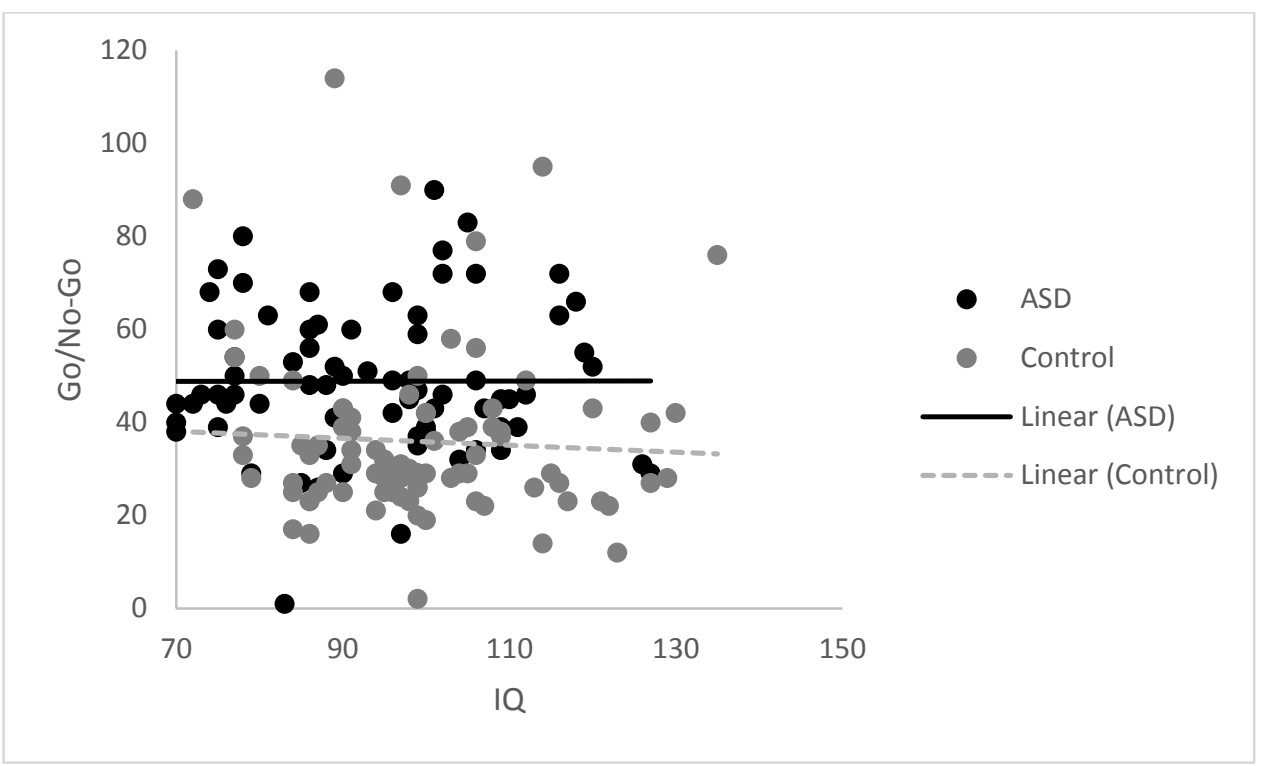

$* p<.05$

Figure 4. 
HOT \& COOL EF TRAJECTORIES IN ASD
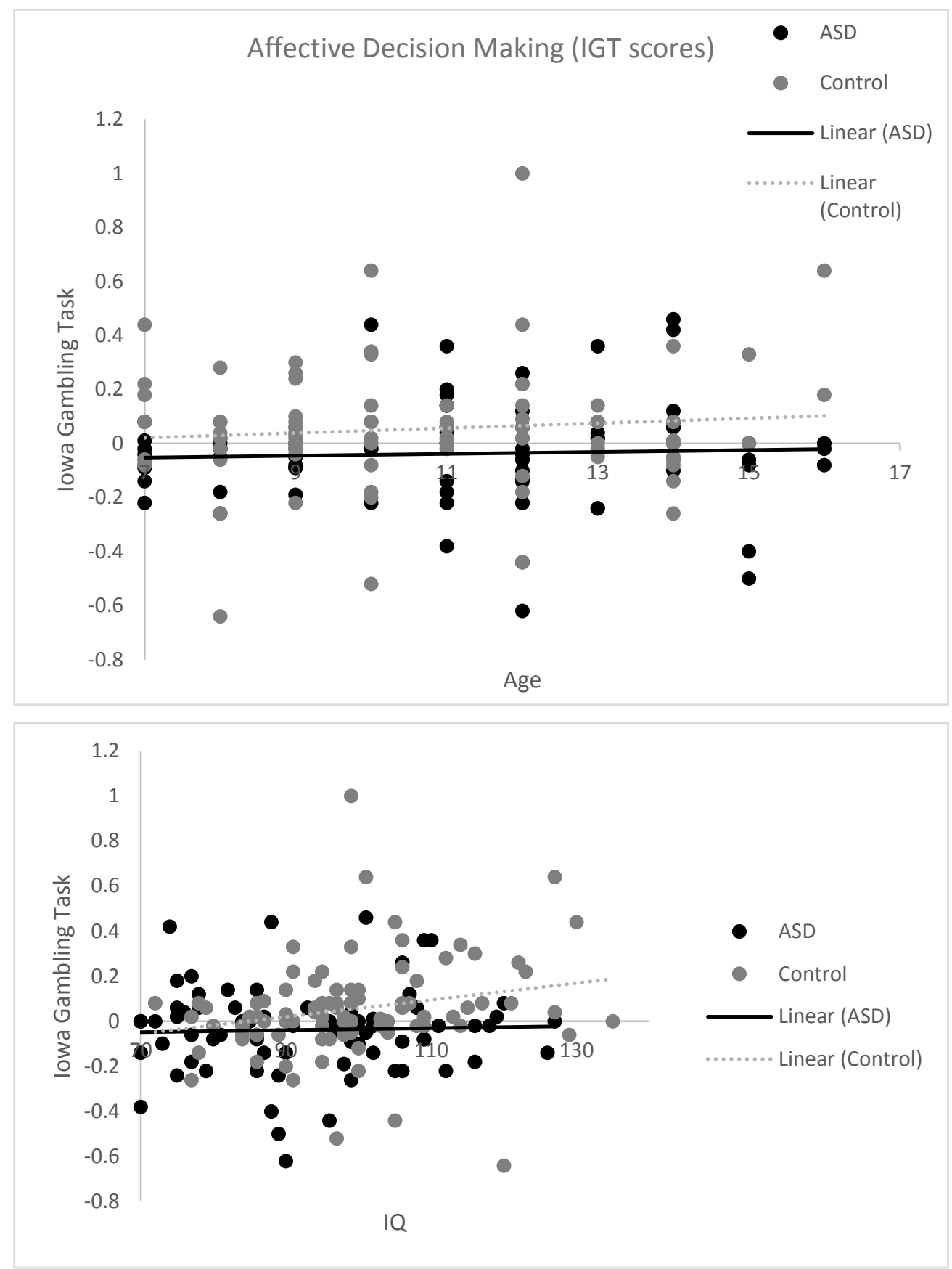

Figure 5. 
HOT \& COOL EF TRAJECTORIES IN ASD
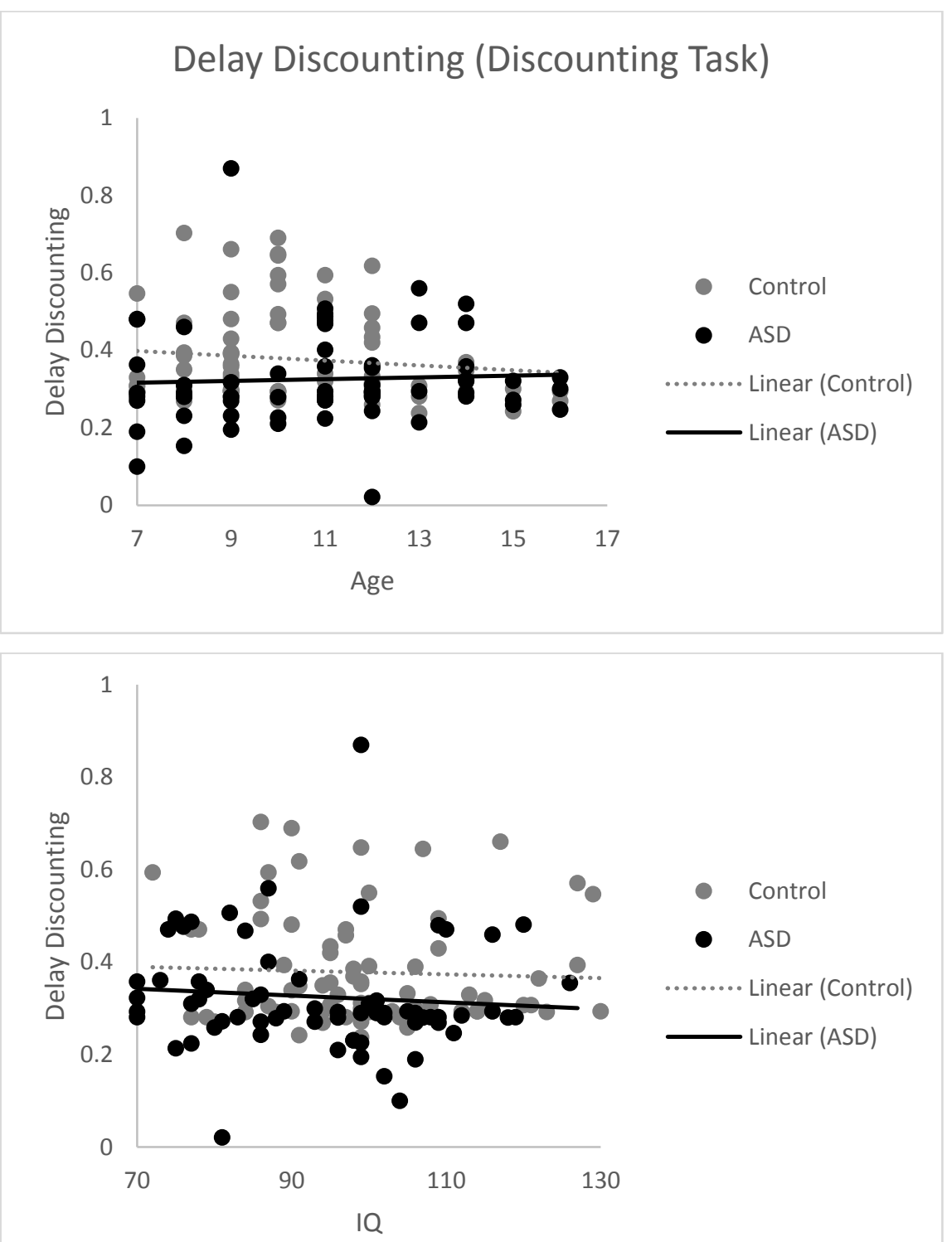